\title{
Progressive ventilation inhomogeneity in infants with cystic fibrosis after pulmonary infection
}

\author{
Shannon J. Simpson ${ }^{1}$, Sarath Ranganathann ${ }^{2,3,4}$, Judy Park ${ }^{1}$, Lidija Turkovic ${ }^{1}$, \\ Roy M. Robins-Browne ${ }^{5}$, Billy Skoric ${ }^{2,3}$, Kathryn A. Ramsey ${ }^{1}$, Tim Rosenow $^{1}$, \\ Georgia L. Banton ${ }^{1}$, Luke Berry ${ }^{1}$, Stephen M. Stick ${ }^{1,6,7}$ and Graham L. Hall ${ }^{1}$, \\ on behalf of AREST CF
}

\begin{abstract}
Affiliations: 'Telethon Kids Institute, University of Western Australia, Subiaco, Australia. ${ }^{2}$ Murdoch Children's Research Institute, Melbourne, Australia. ${ }^{3}$ Department of Respiratory Medicine, Royal Children's Hospital, Melbourne, Australia. "Department of Paediatrics, University of Melbourne, Melbourne, Australia. ${ }^{5}$ Department of Microbiology and Immunology, University of Melbourne, Melbourne, Australia. ${ }^{6}$ Department of Respiratory and Sleep Medicine, Princess Margaret Hospital for Children, Perth, Australia. ${ }^{7}$ School of Paediatrics and Child Health, University of Western Australia, Perth, Australia.
\end{abstract}

Correspondence: Graham Hall, Paediatric Respiratory Physiology, Telethon Kids Institute, University of Western Australia, 100 Roberts Road, Subiaco, WA 6008 Australia. E-mail: graham.hallatelethonkids.org.au

ABSTRACT Measures of ventilation distribution are promising for monitoring early lung disease in cystic fibrosis (CF). This study describes the cross-sectional and longitudinal impacts of pulmonary inflammation and infection on ventilation homogeneity in infants with CF.

Infants diagnosed with CF underwent multiple breath washout (MBW) testing and bronchoalveolar lavage at three time points during the first 2 years of life.

Measures were obtained for 108 infants on 156 occasions. Infants with a significant pulmonary infection at the time of MBW showed increases in lung clearance index (LCI) of 0.400 units (95\% CI 0.150-0.648; $\mathrm{p}=0.002$ ). The impact was long lasting, with previous pulmonary infection leading to increased ventilation inhomogeneity over time compared to those who remained free of infection $(\mathrm{p}<0.05)$. Infection with Haemophilus influenzae was particularly detrimental to the longitudinal lung function in young children with CF where LCI was increased by 1.069 units for each year of life (95\% CI 0.484-1.612; p<0.001).

Pulmonary infection during the first year of life is detrimental to later lung function. Therefore, strategies aimed at prevention, surveillance and eradication of pulmonary pathogens are paramount to preserve lung function in infants with CF.

@ERSpublications

Early life respiratory infections are detrimental to long-term lung function in children with cystic fibrosis http://ow.ly/PKaHn

This article has supplementary material available from erj.ersjournals.com

Received: Sept 232014 | Accepted after revision: June 282015 | First published online: Sept 172015

Support statement: Funding for this study was received from the Department of Health, Australian Government, National Health and Medical Research Council. Funding information for this article has been deposited with FundRef.

Conflict of interest: Disclosures can be found alongside the online version of this article at erj.ersjournals.com

Copyright OERS 2015 


\section{Introduction}

Neutrophil-dominated airway inflammation and infection are the main predictors of structural lung damage and subsequent decline in lung function in cystic fibrosis (CF) [1]. Neutrophils are probably drawn to the lung by a chemotactic protein, interleukin (IL)-8, both of which are elevated in young children with CF [2]. Abnormalities in pulmonary inflammation, endobronchial infection $[3,4]$ and chest computed tomography (CT) [5] have been reported during the first months of postnatal life in infants diagnosed with CF by newborn screening and often in the absence of respiratory symptoms $[6,7]$. Despite these abnormalities, data from the Australian Respiratory Early Surveillance Team for Cystic Fibrosis (AREST CF) have previously shown that infants diagnosed by newborn screening largely have normal expiratory flows and volumes in the first 6 months of life, with lung function deteriorating after this time [8], particularly following a significant pulmonary infection [9]. In contrast, other groups have reported that a significant proportion of infants diagnosed with CF clinically have reduced flows and volumes early in life [10-12].

Measures of lung function have the potential to offer a noninvasive marker of early respiratory dysfunction. Consequently, assessment of the lung clearance index (LCI), a measure of ventilation homogeneity obtained from multiple breath inert gas washout (MBW) has gained popularity over the past decade for monitoring the progression of CF lung disease in the early years $[10,13,14]$. The MBW is a simple to perform, noninvasive test which is ideal for longitudinally monitoring disease since it can be applied from infancy to adulthood [15]. The majority of preschool and school-aged children with CF demonstrate an elevated LCI [16-19], which is more sensitive than spirometry for detecting the presence of lung disease in this age group $[17,20,21]$ and is predictive of lung function outcomes later in childhood $[18,22]$. In addition, the LCI may be a suitable clinical tool for examining the response to therapeutic interventions [23-25], since LCI has been reported to increase with Pseudomonas aeruginosa infection in preschool children [16] and improves following antibiotic therapy [26]; however, the role of LCI in monitoring patients during pulmonary exacerbations remains unclear [27].

While data are limited, the utility of the LCI during infancy in CF is even less clear. Fewer than a quarter of infants diagnosed with CF by newborn screening demonstrate abnormal LCI at 3 months of age [28]. In addition, the presence of bronchiectasis on chest CT [29] or pulmonary infection detected by bronchoalveolar lavage (BAL) [30] show no association with increased LCI during early life in a small population. However, weak associations between elevated LCI and increased markers of airway inflammation are noted [30], as are associations with other indices of ventilation distribution such as moment ratios [29]. As pulmonary inflammation and infection are an important part of the pathophysiology of early CF, the aim of this study was to determine how their presence impacts on ventilation distribution throughout early life. We hypothesised that pulmonary inflammation and infection should result in elevated markers of ventilation homogeneity including the LCI at the time of detection (cross-sectional) and that pulmonary inflammation and infection should impact on subsequent (longitudinal) measures of ventilation distribution, assessed by $\mathrm{MBW}$, in infants diagnosed with CF.

\section{Methods}

\section{Study subjects}

Infants diagnosed with $\mathrm{CF}$ following newborn screening were studied soon after diagnosis (median age 3.6 months), at 12 months and 24 months of age as part of the AREST CF surveillance programme, the full details of which have been published elsewhere [6]. Assessment included infant lung function testing 1-3 days prior to BAL and chest CT. All infants in the AREST CF programme are prescribed amoxicillin-clavulanic acid prophylactically (Augmentin Duo (Aspen Pharmacare Pty Ltd, St Leonards, Australia) $11.25 \mathrm{mg} \cdot \mathrm{kg}^{-1}$ twice daily), and parents were not asked to stop the antibiotic treatment prior to bronchoscopy.

\section{Infant lung function testing}

Infants were studied asleep following oral chloral hydrate $\left(60-100 \mathrm{mg} \cdot \mathrm{kg}^{-1}\right)$. MBW testing was performed using 4\% sulphur-hexafluoride tracer gas and flow was measured using an ultrasonic flowmeter (Ecomedics AG, Duerton, Switzerland) as previously described [31, 32]. A temperature model [33] and deadspace correction were applied to technically acceptable data and the functional residual capacity (FRC), LCI and moment ratios $\left(\mathrm{M}_{1} / \mathrm{M}_{0}\right.$ and $\left.\mathrm{M}_{2} / \mathrm{M}_{0}\right)$ were derived [34, 35]. MBW outcomes from infants with three or more acceptable measurements (FRC within $25 \%$ of median and LCI within one lung turnover) or two acceptable measurements (FRC within $10 \%$ of the highest value and LCI within one lung turnover) were averaged for inclusion in the analysis.

\section{BAL}

BAL was performed when infants were clinically well, as previously published by our group [6]. While acute pulmonary exacerbations are rare in this age group, annual research BALs are routinely delayed until $\sim 6$ weeks after resolution of the exacerbation if these events coincide. Briefly, three aliquots of saline 
$\left(1 \mathrm{~mL} \cdot \mathrm{kg}^{-1}\right)$ were instilled into the right middle lobe, and one aliquot into the lingual or worst-affected lobe on CT, and retrieved using low-pressure suction [36]. Two aliquots (one right and worst) were cultured for the identification of bacteria, fungi and viruses. The remaining two aliquots were stored on ice for assessment of inflammation, which occurred within $3 \mathrm{~h}$.

\section{BAL inflammatory profile}

Inflammatory analyses were performed as previously described $[5,6,37]$ and included assessments of total and differential cell count, IL-8 level by ELISA (BD OptEIA; BD Biosciences, San Diego, CA, USA) and detection of free neutrophil elastase activity using an enzyme activity assay. Inflammatory response scores were derived using a weighted average of the above markers using a principal components analysis [38].

\section{Microbiology}

BAL was cultured on blood, cysteine lactose electrolyte deficient agar, Fildes agar and Sabouraud agar with chloramphenicol. Bacterial and fungal densities were analysed, where $10^{3} \mathrm{cfu} \cdot \mathrm{mL}^{-1}$ is the lowest reported density. A density of $\geqslant 10^{5} \mathrm{cfu} \cdot \mathrm{mL}^{-1}$ with a specific organism is indicative of significant infection with that organism. Positive cultures lacking a predominant organism, largely consisting of organisms expected to be present in the mouth or upper airway, such as oral Neisseria and Streptococcus species, were classified as mixed oral flora (MOF) regardless of density. Pro-inflammatory pathogens were defined as those previously shown to elicit a pro-inflammatory response ( $P$. aeruginosa, Staphylococcus aureus, Haemophilus influenzae, Streptococcus pneumoniae and Aspergillus species) [38].

\section{Clinical characteristics}

Respiratory symptoms were determined by history and examination on the day of the BAL. CF transmembrane conductance regulator (CFTR) genotype was classified as severe (at least one class I, II or III mutation) or mild (class IV or V), as previously described [39-42].

\section{Statistical analysis}

Measures of ventilation inhomogeneity ( $\mathrm{LCI}, \mathrm{M}_{1} / \mathrm{M}_{0}$ and $\mathrm{M}_{2} / \mathrm{M}_{0}$ ) were analysed as continuously distributed outcomes using linear mixed effects models. Associations between these outcomes and pulmonary infection, inflammation, current respiratory symptoms and severe CFTR genotype were determined using separate mixed effects models with random subject effects for each exposure variable and were adjusted for age at BAL. Continuous inflammatory markers were transformed to the natural logarithmic scale to achieve approximate normality. The coefficients of the fixed effects of the linear mixed models with $95 \%$ confidence intervals and p-values are presented.

To investigate the change of ventilation inhomogeneity over time, linear mixed effects models were fitted, assessing the association between measures of ventilation inhomogeneity and presence of pulmonary infections and inflammatory markers (one marker at the time). Models were adjusted for age at BAL and an interaction between infection (or inflammation) marker and age. For simplicity, only the coefficients of interaction effects between age and the presence/absence of infection (or inflammation) are reported, along with the $95 \%$ confidence intervals and p-values. In the longitudinal analyses patients that had a positive BAL at any point in the past were classified as having had a past infection. All analyses were performed using Stata 13.0 (StataCorp, College Station, TX, USA).

\section{Results}

\section{Study population}

Paired measures of ventilation inhomogeneity derived from MBW tests and markers of inflammation and pulmonary infection status were obtained in 108 infants on 156 occasions, with 66 children having one visit, 36 children having two visits and six children having three visits. While most of the 108 infants attended BAL visits at ages $\sim 3$ months, 1 year and 2 years $(n=296$ (91\%) out of an ideal 324 BALs), not all infants had a successful MBW result due to lack of consent for sedation, failure of the infant to sleep after sedation or lack of acceptable, repeatable data $(n=156(53 \%)$ out of a possible 296 visits). There were no differences in age, height, sex or prevalence of inflammatory and infection outcomes between children that had successful MBW and those that did not, with the exception of the presence of pro-inflammatory pathogens ( $26 \%$ in children with MBW compared to $36 \%$ without MBW; $\mathrm{p}=0.05$ ). There were no significant differences in the prevalence of any of the individual pathogens.

Of the 108 children, $89.4 \%$ had a severe genotype (class I, II or III mutations). IL- 8 was present in 125 (85.6\%) BAL samples and free neutrophil elastase was detected in 19 (12.9\%) samples at the time of MBW. Micro-organisms other than MOF were cultured from BAL fluid in 56 (35.9\%) samples at the time of lung function testing, with significant pulmonary infection $\left(\geqslant 10^{5} \mathrm{cfu} \cdot \mathrm{mL}^{-1}\right)$ evident at $25(16 \%)$ visits. The pro-inflammatory pathogens [38] P. aeruginosa (4.5\%), Aspergillus spp. (5.1\%), H. influenzae (7.7\%), 
S. aureus (9.0\%) and S. pneumoniae (2.6\%) were cultured in $28.8 \%$ of the samples at MBW visits. Further demographic and clinical characteristics of the study population can be found in table 1 and a complete infection record is available for each child at the time of BAL in the online supplementary material. There were no differences between sites for any characteristics after adjusting for age at BAL.

Cross-sectional analyses of ventilation inhomogeneity in the presence of inflammation and infection

Pulmonary inflammation

Generally, measures of pulmonary inflammation including total cell and neutrophil counts, presence/ extent of neutrophil elastase and IL-8 were not associated with increased LCI or moment ratios in infants and young children with CF (aged 0-2 years). However, weak associations with the presence of IL-8 $\left(\mathrm{M}_{2} /\right.$ $\mathrm{M}_{0} ; \mathrm{p}=0.045$ ) and total cell count (LCI; $\mathrm{p}=0.030$ ) were evident (table 2).

\section{Pulmonary infection}

Significant pulmonary infection $\left(\geqslant 10^{5} \mathrm{cfu} \cdot \mathrm{mL}^{-1}\right)$ was associated with increased LCI (mean difference 0.400; $\mathrm{p}=0.002$ ) in infants diagnosed with CF following newborn screening (fig. 1 and table 2). Furthermore, infection with Aspergillus spp. or H. influenzae was particularly concomitant with elevation of all measures of ventilation inhomogeneity, while the detection of $P$. aeruginosa, $S$. aureus or mixed oral flora in BAL samples was not associated with increased MBW outcomes (table 2).

\section{Clinical characteristics}

Neither respiratory symptoms, as determined by history and examination on the day of the BAL nor severe genotype were associated with increased LCI or moment ratios (table 2).

\section{Longitudinal analysis of ventilation inhomogeneity in the presence of inflammation and infection Pulmonary inflammation and genotype \\ In general, the direction of the interaction terms in the models suggested a greater decline in lung function over time (increased ventilation inhomogeneity) in those children with a "severe" CFTR genotype (fig. 2c)}

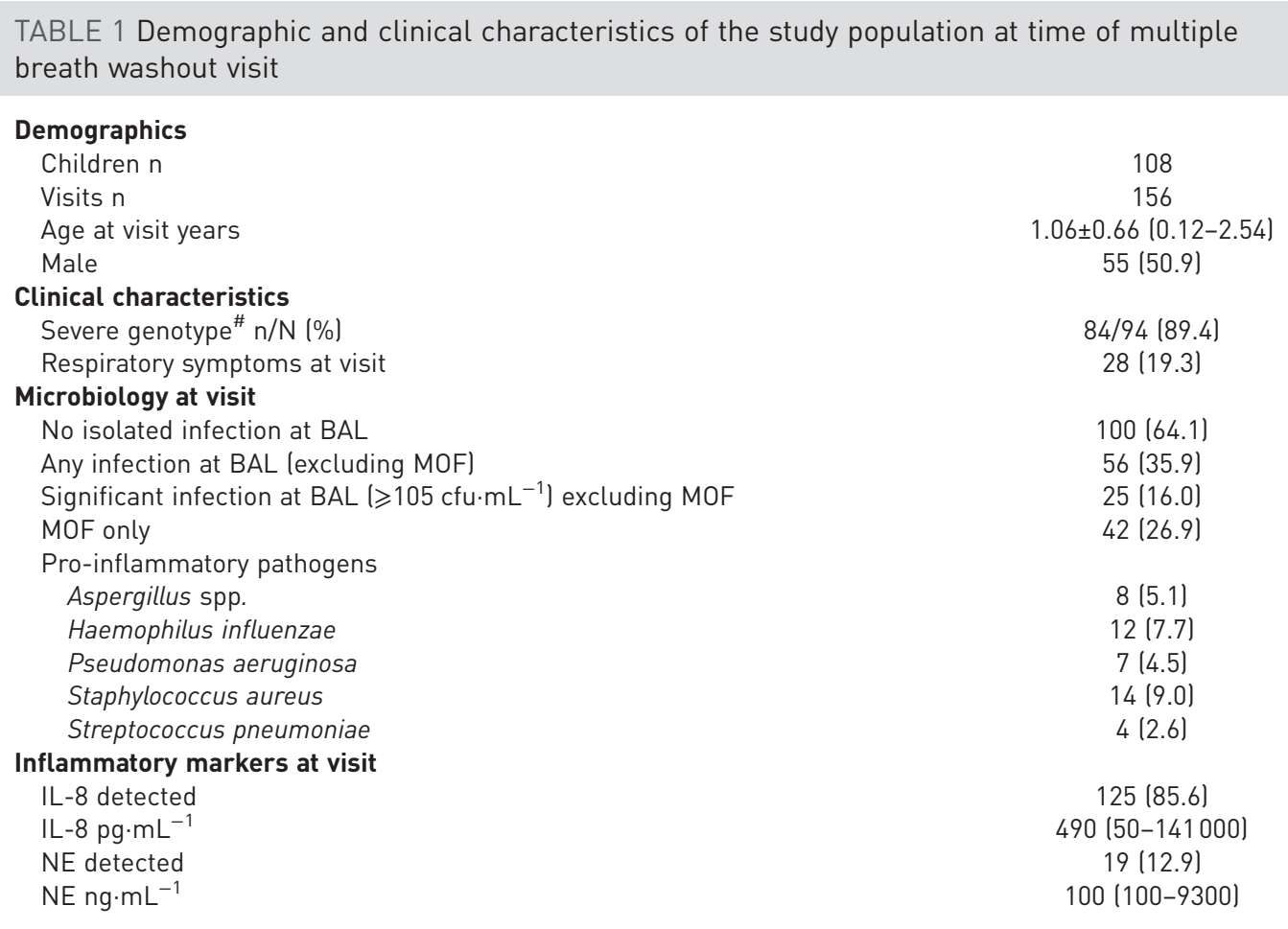

Data are presented as $\mathrm{n}$, mean $\pm \mathrm{SD}$ (range), $\mathrm{n}(\%)$ or median (range), unless otherwise stated. BAL: bronchoalveolar lavage; MOF: mixed oral flora; IL: interleukin; NE: neutrophil elastase. \#: some cystic fibrosis transmembrane conductance regulator (CFTR) mutations have unknown effects on CFTR function and some subjects are yet to have mutations identified on both genes. 
TABLE 2 The effect of current pulmonary infection, inflammation and clinical characteristics on measures of ventilation inhomogeneity in infants

\begin{tabular}{|c|c|c|c|c|c|c|}
\hline & \multicolumn{2}{|l|}{ LCl } & \multicolumn{2}{|l|}{$M_{1} / M_{0}$} & \multicolumn{2}{|l|}{$M_{2} / M_{0}$} \\
\hline & Difference & p-value & Difference & p-value & Difference & p-value \\
\hline \multicolumn{7}{|l|}{ Pulmonary infection } \\
\hline No infection & \multicolumn{2}{|l|}{ Reference } & \multicolumn{2}{|l|}{ Reference } & \multicolumn{2}{|l|}{ Reference } \\
\hline Any infection excluding MOF & $0.211(0.031-0.391)$ & 0.102 & $0.045(-0.007-0.097)$ & 0.089 & $0.331(-0.064-0.725)$ & 0.100 \\
\hline MOF only & $0.125(-0.052-0.302)$ & 0.168 & $-0.003(-0.054-0.048)$ & 0.911 & $-0.008(-0.397-0.381)$ & 0.967 \\
\hline Significant infection excluding MOF $\geq 10^{5} \mathrm{cfu} \cdot \mathrm{mL}^{-1}$ & $0.400(0.150-0.648)$ & 0.002 & $0.121(0.049-0.193)$ & 0.001 & $0.959(0.414-1.504)$ & 0.001 \\
\hline Any infection with a pro-inflammatory pathogen & $0.270(0.063-0.476)$ & 0.011 & $0.070(0.010-0.130)$ & 0.022 & $0.477(0.021-0.932)$ & 0.040 \\
\hline Aspergillus spp. infection & $0.738(0.359-1.112)$ & $<0.001$ & $0.146(0.035-0.257)$ & 0.010 & $1.139(0.295-1.982)$ & 0.008 \\
\hline Haemophilus influenzae infection & $0.483(0.154-0.811)$ & 0.004 & $0.164(0.071-0.258)$ & 0.001 & $1.180(0.456-1.894)$ & 0.001 \\
\hline Pseudomonas aeruginosa infection & $-0.059(-0.479-0.361)$ & 0.783 & $-0.024(-0.144-0.097)$ & 0.700 & $-0.183(-1.098-0.732)$ & 0.695 \\
\hline Staphylococcus aureus infection & $0.054(-0.253-0.360)$ & 0.732 & $0.015(-0.073-0.103)$ & 0.746 & $0.069(-0.601-0.739)$ & 0.840 \\
\hline \multicolumn{7}{|l|}{ Pulmonary inflammation } \\
\hline $\mathrm{TCC} \times 10^{3} \cdot \mathrm{mL}^{-1}$ (for a doubling) & $0.084(-0.008-0.160)$ & 0.030 & $0.011(-0.011-0.033)$ & 0.312 & $0.085(-0.083-0.253)$ & 0.321 \\
\hline Neutrophils $\times 10^{3} \cdot \mathrm{mL}^{-1}$ (for a doubling) & $0.039(-0.007-0.085)$ & 0.095 & $0.002(-0.011-0.016)$ & 0.746 & $0.023(-0.081-0.129)$ & 0.677 \\
\hline NE level (for a doubling) & $0.026(-0.043-0.095)$ & 0.464 & $0.002(-0.018-0.022)$ & 0.845 & $0.028(-0.124-0.179)$ & 0.722 \\
\hline Presence of NE & $-0.017(-0.271-0.237)$ & 0.898 & $0.019(-0.056-0.094)$ & 0.618 & $0.218(-0.334-0.769)$ & 0.439 \\
\hline IL-8 level (for a doubling) & $0.020(-0.006-0.028)$ & 0.228 & $0.007(-0.004-0.019)$ & 0.224 & $0.071(-0.016-0.157)$ & 0.107 \\
\hline Presence of IL-8 & $0.155(-0.085-0.395)$ & 0.206 & $0.064(-0.007-0.134)$ & 0.075 & $0.526(0.012-1.040)$ & 0.045 \\
\hline Inflammatory response score & $0.029(-0.030-0.088)$ & 0.343 & $0.009(-0.009-0.026)$ & 0.303 & $0.078(-0.048-0.204)$ & 0.224 \\
\hline Cumulative mean inflammatory response score & $0.013(-0.062-0.090)$ & 0.729 & $0.006(-0.017-0.028)$ & 0.616 & $0.052(-0.114-0.219)$ & 0.538 \\
\hline \multicolumn{7}{|l|}{ Clinical characteristics } \\
\hline Respiratory symptoms & $0.025(-0.190-0.240)$ & 0.818 & $0.009(-0.054-0.071)$ & 0.790 & $0.083(-0.374-0.541)$ & 0.721 \\
\hline Severe genotype & $0.097(-0.183-0.377)$ & 0.498 & $0.057(-0.030-0.143)$ & 0.199 & $0.438(-0.193-1.070)$ & 0.174 \\
\hline
\end{tabular}




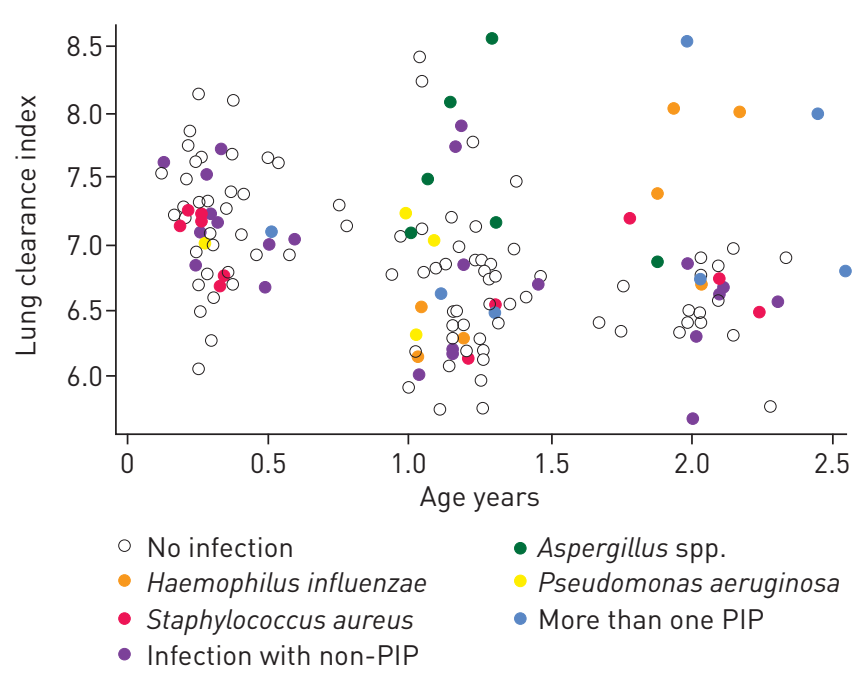

FIGURE 1 Lung clearance index during the early years in cystic fibrosis. Infants are identified as being uninfected lopen circles) or infected with the pro-inflammatory pathogens (PIP) Aspergillus spp., Haemophilus influenzae, Pseudomonas aeruginosa, Staphylococcus aureus, more than one of the above-mentioned PIPs, or any other non-PIP at the time of bronchoalveolar lavage.

or where free neutrophil elastase was detected (fig. 2b). However, the confidence intervals were wide for the estimates of the interaction terms and statistical significance was not reached (table 3 ).

\section{Pulmonary infection}

Infants with $\mathrm{CF}$ who remained free of detectable pulmonary infection in BAL over the first 2 years of life showed a decrease in LCI, $\mathrm{M}_{1} / \mathrm{M}_{0}$ and $\mathrm{M}_{2} / \mathrm{M}_{0}$ during this time (table 4). Children who had ever been identified with a pulmonary infection, either at the time of the visit or at a previous visit, demonstrated accelerated lung function decline (increased ventilation inhomogeneity) when compared to those who had never been identified with a pulmonary infection (fig. 2 and table 3). The increase in ventilation
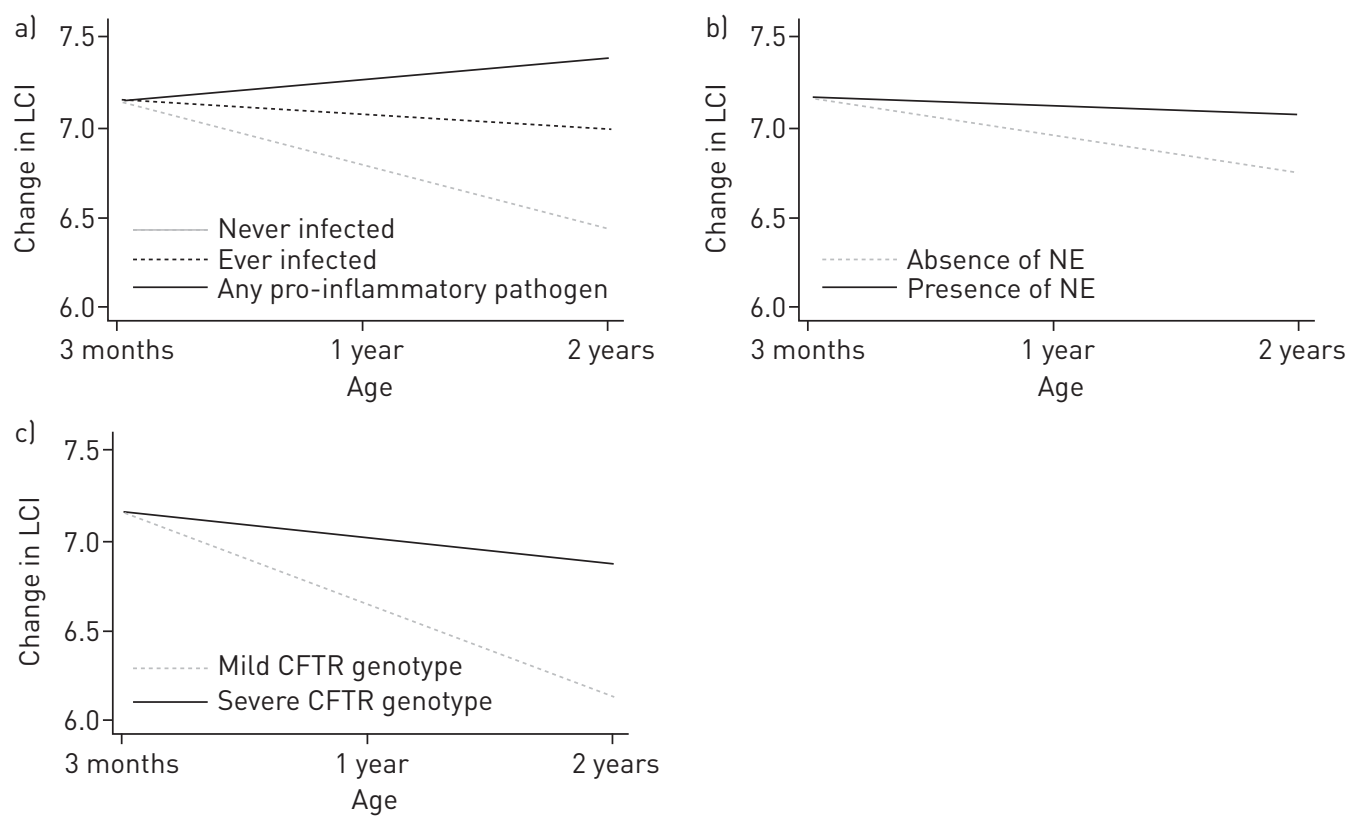

FIGURE 2 The change in lung clearance index (LCI) over the first 2 years of life in children with cystic fibrosis. Linear mixed effects models were used to predict the association between $\mathrm{LCl}$ and a) the presence of pulmonary pathogens; b) the presence of neutrophil elastase (NE); and cl severe cystic fibrosis transmembrane conductance regulator (CFTR) genotype over time from the median LCl at 3 months of age. The solid lines represent the predicted values for those children who al remained free of pulmonary pathogens in the first 2 years of life; b) had no free NE detected; and c) were classified as having a mild genotype. 
TABLE 3 The longitudinal effects of pulmonary infection and inflammation on measures of ventilation inhomogeneity in infancy

LCI

$M_{1} / M_{0}$

$M_{2} / M_{0}$

Interaction between infection p-value status and age $(95 \% \mathrm{CI})$

Interaction between infection status and age $(95 \% \mathrm{CI})$

p-value

Interaction between infection

p-value ${ }^{\#}$

Reference

Never infected

Current infection and no past infection

Current infection and past infection

Reference

$0.361(-0.06-0.768)$

Reference

$0.656(0.180-1.132) \quad \mathbf{0 . 0 0 7} \quad 0.181(0.044-0.317)$

$0.699(0.061-1.337) \quad \mathbf{0 . 0 3 2} \quad 0.243(0.060-0.426)$

Infection with any pro-inflammatory

$0.449(0.160-0.738)$

$0.124(0.041-0.207)$

pathogen

Aspergillus spp.

Haemophilus influenzae

Pseudomonas aeruginosa

$-0.044(-0.619-0.531)$

$1.069(0.548-1.591)$

0.002

$0.124(0.041-0.207)$

Staphylococcus aureus

$0.087(-0.561-0.736)$

$-0.036(-0.221-0.150)$

Absence of free NE

$0.343(-0.016-0.702)$

0.001
0.792

Reference

0.061

$0.076(-0.028-0.179)$ Reference

Presence of free NE

Absence of IL-8

0.204 (-0.125-0.533)

0.225

$0.042(-0.053-0.136)$

Reference

Presence of IL-8

$0.066(-0.432-0.564)$ Reference

Non-severe genotype

Reference

0.796

$0.021(-0.123-0.165)$

Reference

Severe genotype

0.101

0.134 (0.009-0.258) status and age $(95 \% \mathrm{Cl})$

Data represent the interaction effects between age and infection status on lung function (lung clearance index (LCI), 1st to 0th moment ratio ( $\mathrm{M}_{1} / \mathrm{M}_{0}$ ) and 2 nd to 0 th $\mathrm{moment}$ ratio ( $\mathrm{M}_{2}$ / Moll. Data presented in bold are statistically significant. Longitudinal infection variables take into account all microbiology data available prior to the last multiple breath washout (MBW) visit, even for the visits where MBW was not performed/successful and therefore "never infected" subjects are children that were not infected with any pathogen including mixed oral flora at the current bronchoalveolar lavage (BAL) and all past BALs. See the online supplementary material for further infection information. NE: neutrophil elastase; IL: interleukin.

: $p$-value for the interaction between infection status and age. 


\begin{tabular}{|c|c|c|c|}
\hline & $<6$ months & 6-18 months & $>18$ months \\
\hline Subjects $n / N$ & $23 / 49$ & $12 / 72$ & $3 / 35$ \\
\hline $\mathrm{LCl}$ & $7.15 \pm 0.51$ & $6.79 \pm 0.70$ & $6.36 \pm 0.05$ \\
\hline$M_{1} / M_{0}$ & $2.09 \pm 0.17$ & $1.96 \pm 0.19$ & $1.83 \pm 0.09$ \\
\hline$M_{2} / M_{0}$ & $7.79 \pm 1.23$ & $6.87 \pm 1.38$ & $5.88 \pm 0.70$ \\
\hline
\end{tabular}

Data are presented as mean $\pm S D$, unless otherwise stated. $\mathrm{LCl}$ : lung clearance index; $\mathrm{M}_{1} / \mathrm{M}_{0}$ : 1 st to 0 th moment ratio; $M_{2} / M_{0}$ : 2 nd to 0 th moment ratio.

inhomogeneity was particularly apparent if one of the bacterial pathogens known to elicit a pro-inflammatory response ( $P$. aeruginosa, S. aureus, H. influenzae, S. pneumoniae and Aspergillus species) [38] was isolated from the lower respiratory tract, with a mean increase of 0.449 LCI units for every 1 year after the isolation (fig. 2a). The greatest effects were associated with isolation of $H$. influenzae (1.069 LCI units; $\mathrm{p}<0.001$ ). Infants who were free from any detectable infection at the time of testing, but who had previously exhibited a positive bacterial culture recorded a mean increase of 0.699 LCI units per year compared to those who remained uninfected.

\section{Discussion}

This study demonstrates that significant pulmonary infection is associated with increased ventilation inhomogeneity, measured using inert gas washout in infants diagnosed with CF by newborn screening. A longitudinal assessment of our unique dataset demonstrated the impact of pulmonary inflammation and infection on ventilation distribution in infants with CF over time. Children with prior pulmonary infection exhibited accelerated lung function decline (increase in LCI, $M_{1} / M_{0}$ and $M_{2} / M_{0}$ ) when compared to those infants who remained free of detectable pulmonary pathogens over the first 2 years of life. Increased ventilation inhomogeneity was marked in the presence of infection with those pathogens previously shown to elicit a strong inflammatory response [38], and in particular infection with $H$. influenzae significantly impacted on the longer-term lung function in young children with CF.

The LCI as an outcome measure for the detection of early CF lung disease has been supported by a number of studies that demonstrate that LCI is increased, even in the presence of a normal forced expiratory volume in $1 \mathrm{~s}$, in young school-aged children $[16,43]$. However, the role of LCI in CF during infancy remains unclear, with $>80 \%$ infants with CF reporting an LCI within normal limits at 3 months of age which increases with increasing age $[10,30]$. Reference values of LCI tend to be higher in infancy than in older children [15, 16], with the upper limit of normal defined as 8.3 in 3-month-old infants [44]. We report that none of the 3-month-old infants in this study demonstrated an LCI $>8.3$ regardless of infection or inflammation status. An important caveat is that the reference data were collected on different, non-commercially available, equipment and therefore may not be directly comparable to this population and that adequate reference values for data using commercially available equipment suitable for measurement in infants are not currently available.

The LCI is potentially a suitable clinical tool for surveillance of disease, detecting pulmonary exacerbations and examining the response to therapeutic intervention in older children with CF $[23,24,45]$. In this study we have shown that significant pulmonary infection is associated with increased LCI during infancy (mean difference 0.400 LCI units), which is an important first step to demonstrate the surveillance and intervention potential of this technique during the early years of life. The LCI would be a particularly attractive clinical trial end-point during infancy, since the MBW is relatively simple to perform, unlike other infant lung function tests such as the raised volume rapid thoracic compression technique, and is comparatively less invasive than BAL. However, beyond the first few months of life, sedation of the subject is still required. A sensitive, reproducible and feasible clinical trial end-point that can be used during infancy is crucial for trials of disease-modifying therapies that are intended for lifelong use commencing soon after diagnosis.

We report worse outcomes (increase in LCI, $\mathrm{M}_{1} / \mathrm{M}_{0}$ and $\mathrm{M}_{2} / \mathrm{M}_{0}$ ) in children at 2 years of age where a pulmonary infection was ever isolated, even in the absence of a concurrent infection at the time of testing. Infection with $H$. influenzae was particularly detrimental to the respiratory health of young children with $\mathrm{CF}$, with an increase of $1.069 \mathrm{LCI}$ units for each year after its isolation in BAL when compared to those who remained free of pulmonary infection. While a common isolate in young children with CF, the specific role that $H$. influenzae plays in the development or progression of early CF lung disease currently remains unclear. However, this study in addition to our recent publication showing that infection with 
H. influenzae in early life leads to reduced spirometry at school age [46] provides some evidence that early-life infection with $H$. influenzae may worsen the prognosis of the disease. Such detriment may be attributed to the genetic diversity of $\mathrm{H}$. influenzae, with some isolates from young children with $\mathrm{CF}$ proving to be strong biofilm producers [47]. In light of our findings, further studies would be helpful to determine whether antibiotic prophylaxis or the development of specific eradication protocols directed against the other pro-inflammatory pathogens such as $H$. influenzae and S. aureus [48] are worth pursuing in order to limit decline in lung function in early life. With the exception of $P$. aeruginosa, current protocols for the management of early CF would not necessarily lead to antibiotic or antifungal treatment after the detection of these pathogens, especially in asymptomatic individuals [49].

We were unable to elucidate the effect of antibiotic prophylaxis on bacterial profiles in BAL and impact on longitudinal lung function as all of the infants in the AREST CF programme are prescribed prophylactic antibiotics and no data on adherence were available. As a result of prophylactic antibiotic use, it is also possible that the bacterial profiles described in our cohort may differ to other centres where the use of prophylactic antibiotics in CF does not form part of routine clinical care.

Our group has previously shown that pulmonary infection is associated with a greater rate of lung function decline by forced expiratory flows and volumes in a newborn screening population [7], and it should be noted that a small subset of infants are common to both studies. While there is evidence to suggest that reduced lung function by spirometry tracks from infancy into the preschool years [50] and that LCI measured at 3-5 years predicts subsequent abnormal lung function during the early school years [18] in those clinically diagnosed with CF, it remains speculation that those with faster declining lung function in infancy have poorer clinical outcomes later in life.

While it is known that pulmonary infection results in an excessive inflammatory response in CF airways $[38,51]$, the possible role of inflammation as an independent contributor to early CF pathophysiology has also been established $[4,52]$. In the current study, the presence of IL-8 or neutrophil elastase was not significantly associated with accelerated lung function decline. This may in part be due to the limited number of specific areas of the lung that were sampled for inflammatory analysis in this study, with the right middle lobe or worst affected lobe being used for microbiology rather than inflammation analyses. In addition, only $13 \%$ of the population had detectable levels of free neutrophil elastase, while $86 \%$ had detectable IL-8 levels. Alternatively, since inflammation is known to increase with age regardless of the presence of pulmonary infections in CF [53], the relatively low levels in infancy may not have reached some "critical point" whereby they impact upon ventilation distribution. These data suggest that the functional measurement of MBW is less sensitive than the structural disease observed on chest CT as a measure of the effects of inflammation on lung structure during infancy.

This study demonstrated increased ventilation inhomogeneity (moment ratios) over time in infants with severe CFTR genotype compared to those with mild genotype. The impact of genotype on the severity of lung disease is complicated by the influence of environmental and secondary genetic factors, although there is some evidence to suggest that patients with mild CFTR mutations show reduced rates and delayed acquisition of pulmonary infections [54] as well as less severe progression of pulmonary disease than those patients carrying severe genotypes (class I, II and III CFTR mutations) [55-57].

While the present study may be criticised for the lack of healthy control infants, it was our intention to determine the impact of pulmonary infection and inflammation on measures of ventilation distribution in infants who have early CF lung disease. We therefore do not believe that this design feature alters the significance of the observations, but does highlight the need for healthy reference ranges derived from measurements on commercially available equipment in this age group [58]. We recognise that without the longitudinal assessment of healthy infants we remain unable to comment on whether children with CF remaining free of pulmonary infection experience increased ventilation inhomogeneity over time. Additionally, these data are limited by a lack of information available regarding any possible pulmonary exacerbations between BAL visits, including viral exacerbations. While acute pulmonary exacerbations are relatively rare among young infants, we do not have measures between BAL samples; in part because there is evidence to suggest that the micro-organisms present in the upper airway do not adequately reflect the lower airway [59].

Our findings support the notion that acquisition of any pulmonary infection within the first year of life is potentially a critical factor in predicting long-term lung function decline in children with CF. Therefore, early intervention such as antimicrobial prophylaxis, surveillance for pulmonary infections and the eradication of isolated pathogens may benefit the clinical management of patients with CF during early life. As any infection, even if subsequently eradicated, seems to be associated with persisting decline in lung function, better targeting of the underlying pathology, or other features consequent to the basic biochemical defect in CF [60] are perhaps also warranted in infants with CF. In addition, the data 
presented here provide evidence that measures of ventilation inhomogeneity may offer potential as a suitable outcome for use in clinical trials in infants with CF. Although CT is the current gold standard for demonstrating small structural changes and early progression of lung disease, CT is not a feasible outcome measure for frequent monitoring of disease status. Our data demonstrate that the LCI is affected by the same pathobiological factors that are associated with progression of structural lung disease and therefore might be useful for longitudinal monitoring of lung disease severity.

\section{Acknowledgements}

The authors would like to acknowledge those clinical fellows/researchers who contributed to the collection of bronchoscopy and lung function data and the microbiology departments at the Royal Children's Hospital (Parkville, Australia) and the Princess Margaret Hospital (Subiaco, Australia) as well as the Australian Respiratory Early Surveillance Team for Cystic Fibrosis (AREST CF) families.

\section{References}

1 Konstan MW, Hilliard KA, Norvell TM, et al. Bronchoalveolar lavage findings in cystic fibrosis patients with stable, clinically mild lung disease suggest ongoing infection and inflammation. Am J Respir Crit Care Med 1994; 150: 448-454.

2 Muhlebach MS, Stewart PW, Leigh MW, et al. Quantitation of inflammatory responses to bacteria in young cystic fibrosis and control patients. Am J Respir Crit Care Med 1999; 160: 186-191.

3 Dakin CJ, Numa AH, Wang $\mathrm{H}$, et al. Inflammation, infection, and pulmonary function in infants and young children with cystic fibrosis. Am J Respir Crit Care Med 2002; 165: 904-910.

4 Armstrong DS, Grimwood K, Carlin JB, et al. Lower airway inflammation in infants and young children with cystic fibrosis. Am J Respir Crit Care Med 1997; 156: 1197-1204.

5 Stick SM, Brennan S, Murray C, et al. Bronchiectasis in infants and preschool children diagnosed with cystic fibrosis after newborn screening. J Pediatr 2009; 155: 623-628.

6 Sly PD, Brennan S, Gangell C, et al. Lung disease at diagnosis in infants with cystic fibrosis detected by newborn screening. Am J Respir Crit Care Med 2009; 180: 146-152.

7 Mott LS, Gangell CL, Murray CP, et al. Bronchiectasis in an asymptomatic infant with cystic fibrosis diagnosed following newborn screening. J Cyst Fibros 2009; 8: 285-287.

8 Linnane BM, Hall GL, Nolan G, et al. Lung function in infants with cystic fibrosis diagnosed by newborn screening. Am J Respir Crit Care Med 2008; 178: 1238-1244.

9 Pillarisetti N, Williamson E, Linnane B, et al. Infection, inflammation, and lung function decline in infants with cystic fibrosis. Am J Respir Crit Care Med 2011; 184: 75-81.

10 Lum S, Gustafsson P, Ljungberg $\mathrm{H}$, et al. Early detection of cystic fibrosis lung disease: multiple-breath washout versus raised volume tests. Thorax 2007; 62: 341-347.

11 Ranganathan SC, Dezateux C, Bush A, et al. Airway function in infants newly diagnosed with cystic fibrosis. Lancet 2001; 358: 1964-1965.

12 Ranganathan SC, Stocks J, Dezateux C, et al. The evolution of airway function in early childhood following clinical diagnosis of cystic fibrosis. Am J Respir Crit Care Med 2004; 169: 928-933.

13 Aurora P. Multiple-breath inert gas washout test and early cystic fibrosis lung disease. Thorax 2010; 65: 373-374.

14 Robinson PD, Lindblad A, Gustafsson PM. Comparison of the utility of multiple breath inert gas washout parameters in cystic fibrosis. Thorax 2010; 65: 659.

15 Aurora P, Kozlowska W, Stocks J. Gas mixing efficiency from birth to adulthood measured by multiple-breath washout. Respir Physiol Neurobiol 2005; 148: 125-139.

16 Aurora P, Bush A, Gustafsson P, et al. Multiple-breath washout as a marker of lung disease in preschool children with cystic fibrosis. Am J Respir Crit Care Med 2005; 171: 249-256.

17 Gustafsson PM, De Jong PA, Tiddens HA, et al. Multiple-breath inert gas washout and spirometry versus structural lung disease in cystic fibrosis. Thorax 2008; 63: 129-134.

18 Aurora P, Stanojevic S, Wade A, et al. Lung clearance index at 4 years predicts subsequent lung function in children with cystic fibrosis. Am J Respir Crit Care Med 2011; 183: 752-758.

19 Owens CM, Aurora P, Stanojevic S, et al. Lung Clearance Index and HRCT are complementary markers of lung abnormalities in young children with CF. Thorax 2011; 66: 481-488.

20 Kraemer R, Blum A, Schibler A, et al. Ventilation inhomogeneities in relation to standard lung function in patients with cystic fibrosis. Am J Respir Crit Care Med 2005; 171: 371-378.

21 Ellemunter H, Fuchs SI, Unsinn KM, et al. Sensitivity of Lung Clearance Index and chest computed tomography in early CF lung disease. Respir Med 2010; 104: 1834-1842.

22 Kieninger E, Singer F, Fuchs O, et al. Long-term course of lung clearance index between infancy and school-age in cystic fibrosis subjects. J Cyst Fibros 2011; 10: 487-490.

23 Amin R, Subbarao P, Lou W, et al. The effect of dornase alfa on ventilation inhomogeneity in patients with cystic fibrosis. Eur Respir J 2011; 37: 806-812.

24 Amin R, Subbarao P, Jabar A, et al. Hypertonic saline improves the LCI in paediatric patients with CF with normal lung function. Thorax 2010; 65: 379-383.

25 Sly PD, Brennan S. Detecting early lung disease in cystic fibrosis: are current techniques sufficient? Thorax 2004; 59: 1008-1010.

26 Robinson PD, Cooper P, Van Asperen P, et al. Using index of ventilation to assess response to treatment for acute pulmonary exacerbation in children with cystic fibrosis. Pediatr Pulmonol 2009; 44: 733-742.

27 Welsh L, Nesci C, Tran H, et al. Lung clearance index during hospital admission in school-age children with cystic fibrosis. J Cyst Fibros 2014; 13: 687-691.

28 Hoo AF, Thia LP, Nguyen TT, et al. Lung function is abnormal in 3-month-old infants with cystic fibrosis diagnosed by newborn screening. Thorax 2012; 67: 874-881.

29 Hall GL, Logie KM, Parsons F, et al. Air trapping on chest CT is associated with worse ventilation distribution in infants with cystic fibrosis diagnosed following newborn screening. PLoS One 2011; 6: e23932. 
Belessis Y, Dixon B, Hawkins G, et al. Early cystic fibrosis lung disease detected by bronchoalveolar lavage and lung clearance index. Am J Respir Crit Care Med 2012; 185: 862-873.

Schibler A, Hall GL, Businger F, et al. Measurement of lung volume and ventilation distribution with an ultrasonic flow meter in healthy infants. Eur Respir J 2002; 20: 912-918.

Schulzke SM, Hall GL, Nathan EA, et al. Lung volume and ventilation inhomogeneity in preterm infants at 15-18 months corrected age. J Pediatr 2010; 156: 542-549.

Latzin P, Sauteur L, Thamrin C, et al. Optimized temperature and deadspace correction improve analysis of multiple breath washout measurements by ultrasonic flowmeter in infants. Pediatr Pulmonol 2007; 42: 888-897.

Saidel GM, Salmon RB, Chester EH. Moment analysis of multibreath lung washout. J Appl Physiol 1975; 38: 328-334.

Robinson PD, Goldman MD, Gustafsson PM. Inert gas washout: theoretical background and clinical utility in respiratory disease. Respiration 2009; 78: 339-355.

de Blic J, Midulla F, Barbato A, et al. Bronchoalveolar lavage in children. ERS Task Force on bronchoalveolar lavage in children. Eur Respir J 2000; 15: 217-231.

Brennan S, Hall GL, Horak F, et al. Correlation of forced oscillation technique in preschool children with cystic fibrosis with pulmonary inflammation. Thorax 2005; 60: 159-163.

Gangell C, Gard S, Douglas T, et al. Inflammatory responses to individual microorganisms in the lungs of children with cystic fibrosis. Clin Infect Dis 2011; 53: 425-432.

Rowntree RK, Harris A. The phenotypic consequences of CFTR mutations. Ann Hum Genet 2003; 67: 471-485.

Green DM, McDougal KE, Blackman SM, et al. Mutations that permit residual CFTR function delay acquisition of multiple respiratory pathogens in CF patients. Respir Res 2010; 11: 140

Zielenski J. Genotype and phenotype in cystic fibrosis. Respiration 2000; 67: 117-133.

Zielenski J, Tsui LC. Cystic fibrosis: genotypic and phenotypic variations. Annu Rev Genet 1995; 29: 777-807.

Gustafsson PM, Aurora P, Lindblad A. Evaluation of ventilation maldistribution as an early indicator of lung disease in children with cystic fibrosis. Eur Respir J 2003; 22: 972-979.

Hoo A, Chudleigh J, Prasad A, et al. Is lung function diminished by 3 months of age in infants with cystic fibrosis (CF) diagnosed by newborn screening (NBS)? Eur Respir J 2010; 36: Suppl. 54, 1016 s.

Davies J, Sheridan H, Bell N, et al. Assessment of clinical response to ivacaftor with lung clearance index in cystic fibrosis patients with a G551D-CFTR mutation and preserved spirometry: a randomised controlled trial. Lancet Respir Med 2013; 1: 630-638.

Ramsey KA, Ranganathan S, Park J, et al. Early respiratory infection is associated with reduced spirometry in children with cystic fibrosis. Am J Respir Crit Care Med 2014; 190: 1111-1116.

Starner TD, Zhang N, Kim G, et al. Haemophilus influenzae forms biofilms on airway epithelia: implications in cystic fibrosis. Am J Respir Crit Care Med 2006; 174: 213-220.

Wong JK, Ranganathan SC, Hart E. Staphylococcus aureus in early cystic fibrosis lung disease. Pediatr Pulmonol 2013; 48: 1151-1159.

Borowitz D, Robinson KA, Rosenfeld M, et al. Cystic Fibrosis Foundation evidence-based guidelines for management of infants with cystic fibrosis. J Pediatr 2009; 155: Suppl. 6, S73-S93.

Kozlowska WJ, Bush A, Wade A, et al. Lung function from infancy to the preschool years after clinical diagnosis of cystic fibrosis. Am J Respir Crit Care Med 2008; 178: 42-49.

Armstrong DS, Grimwood K, Carzino R, et al. Lower respiratory infection and inflammation in infants with newly diagnosed cystic fibrosis. BMJ 1995; 310: 1571-1572.

Khan TZ, Wagener JS, Bost T, et al. Early pulmonary inflammation in infants with cystic fibrosis. Am J Respir Crit Care Med 1995; 151: 1075-1082.

Douglas TA, Brennan S, Gard S, et al. Acquisition and eradication of P. aeruginosa in young children with cystic fibrosis. Eur Respir J 2009; 33: 305-311.

4 Green DM, McDougal KE, Blackman SM, et al. Mutations that permit residual CFTR function delay acquisition of multiple respiratory pathogens in CF patients. Respir Res 2010; 11: 140

Corey M, Edwards L, Levison $\mathrm{H}$, et al. Longitudinal analysis of pulmonary function decline in patients with cystic fibrosis. J Pediatr 1997; 131: 809-814.

6 Gan KH, Geus WP, Bakker W, et al. Genetic and clinical features of patients with cystic fibrosis diagnosed after the age of 16 years. Thorax 1995; 50: 1301-1304.

Gan $\mathrm{KH}$, Veeze HJ, van den Ouweland AM, et al. A cystic fibrosis mutation associated with mild lung disease. N Engl J Med 1995; 333: 95-99.

Peterson-Carmichael SL, Rosenfeld M, Ascher SB, et al. Survey of clinical infant lung function testing practices. Pediatr Pulmonol 2014; 49: 126-131.

9 Goddard AF, Staudinger BJ, Dowd SE, et al. Direct sampling of cystic fibrosis lungs indicates that DNA-based analyses of upper-airway specimens can misrepresent lung microbiota. Proc Natl Acad Sci USA 2012; 109: 13769-13774.

60 Cohen TS, Prince A. Cystic fibrosis: a mucosal immunodeficiency syndrome. Nat Med 2012; 18: 509-519. 